\title{
Archéopages Archéopages
}

Archéologie et société

Hors-série 3 | 2012

Nouveaux champs de la recherche archéologique

\section{Processus politiques de déromanisation dans l'entité gothe des Gaules, le regnum tolosanum (413-508)}

Jean-Luc Boudartchouk

\section{CpenEdition}

Journals

Édition électronique

URL : https://journals.openedition.org/archeopages/713

DOI : $10.4000 /$ archeopages.713

ISSN : 2269-9872

Éditeur

INRAP - Institut national de recherches archéologiques préventives

Édition imprimée

Date de publication : 1 janvier 2012

Pagination : 158-165

ISSN : 1622-8545

Référence électronique

Jean-Luc Boudartchouk, «Processus politiques de déromanisation dans l'entité gothe des Gaules, le regnum tolosanum (413-508) », Archéopages [En ligne], Hors-série 3 | 2012, mis en ligne le 01 janvier 2012, consulté le 25 février 2023. URL : http://journals.openedition.org/archeopages/713 ; DOI : https://doi.org/10.4000/archeopages.713 


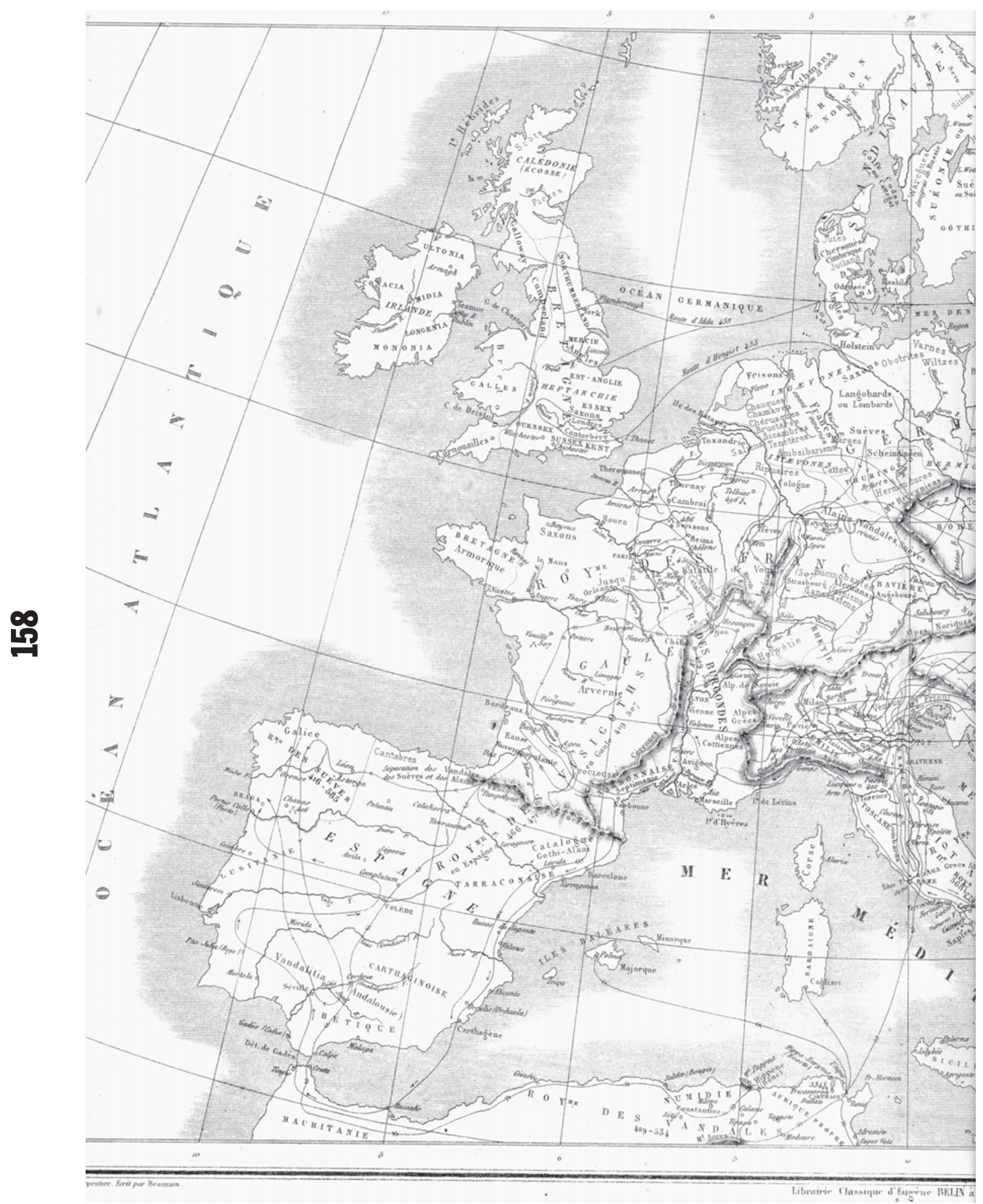

[Fig. 1] Drioux et Leroy, 1875. Carte intitulée : « Europe pour l'invasion des barbares (...) 395-582». Les trajets des différents peuples, reconstitués grâce aux sources écrites, sont représentés par autant de flèches ; les Wisigoths, selon la carte, franchissent le Rhône en provenance d'Italie, atteignent Narbonne, Toulouse, Bazas,

Bordeaux, puis passent en Espagne.

La partie gauloise du royaume des

Goths, que l'on indique ici fondée en

419, y est circonscrite par la Loire et

le Rhône, la Méditerranée, les

Pyrénées et l'Océan : des frontières

naturelles, les mêmes qui sont

énumérées par l'historien

mérovingien Frédégaire. 
devrait permettre de répondre à de multiples questions encore aujourd'hui en suspend. La date de la fondation du site de Moulay, le statut et le rôle de l'espace de l'enceinte de 12 hectares sont encore difficile à appréhender. La nouvelle enceinte de l'oppidum pourrait correspondre à une vaste ville nouvelle qui se serait développée $a$ priori $\mathrm{au} \mathrm{I}^{\mathrm{er}}$ siècle avant notre ère au pied l'espace fortifié restreint. L'ensemble du site est par ailleurs abandonné à l'époque romaine. La compréhension de l'organisation générale de la ville et de son urbanisation demeure un objectif majeur de l'étude en cours. L'étendue et l'essor rapide de la ville nouvelle pourraient témoigner de la puissance de l'élite locale. La planification de l'espace urbain qui transparait semble renvoyer à une administration forte et complexe, capable de rassembler les corps de métier nécessaires à sa mise en œuvre. Une affaire à suivre...

BOISSEL R., DIEHL R., NAVEAU J., 1972 : «L'oppidum de Moulay (fouilles de l'été 1972) », Bulletin de la Commission historique et archéologique de la Mayenne, 243, p. 3-36.

Fichtl S., 2000 (rééd. 2005), La ville celtique. Les oppida de 150 av. J.-C. à 15 ap. J.-C., Paris, Errance, 238 p.

Guichard V., 2007 (octobre), «Les oppida: Consensus autour d'un phénomène hétérogène », in Archéopages, n 20, p. 6-11.

LE GOFF E., 2007, « Découverte d'un habitat groupé de la fin de la période gauloise chez les Osismes : Nouvelles perśpectives pour aborder l'occupation du sol et la structuration du territoire laténien du secteur de Quimper ", AREMORICA, Études sur l'ouest de la Gaule romaine, $\mathrm{n}^{\circ} 1$, Centre de Recherche Bretonne et Celtique, Université de Bretagne Occidentale, Brest, p. 9-14.

LE Goff E., 2008 (août-septembre), « L'agglomération gauloise de Kergolvez à Quimper ", L’Archéologue/Archéologie nouvelle, n 97, p. 23.

NAVEAU J., 1973 : «L'oppidum de Moulay : fouilles de l'année 1973 », Bulletin de la Commission historique et archéologique de la Mayenne, 244, p. 5-17.

Naveau J., 1974 : «L'oppidum de Moulay : fouilles de l'année 1974 », Bulletin de la Commission historique et archéologique de la Mayenne, 245, p. 3-35.

NAVEAU J., 1975-1976 : « L'oppidum de Moulay (travaux de l'année 1975)», Bulletin de la Commission historique et archéologique de la Mayenne, 246-247, p. 61-98

NAVEAU J., 1998, « Le chasseur, l'agriculteur et l'artisan », Guide du musée archéologique de Jublains, Conseil général de la Mayenne, Laval, Service départemental de l'archéologie et des musées, 176 p.

\section{Processus politiques de déromanisation dans l'entité gothe des Gaules, le regnum tolosanum (413-508)}

Jean-Luc Boudartchouk

Inrap, UMR 5608 TRACES

L histoire et l'archéologie du « royaume de Toulouse » restent toujours, dans une large mesure, à explorer [Fig.1]. Nul engouement comparable à celui qui a prévalu, depuis le XVII ${ }^{\mathrm{e}}$ siècle, au sujet des Mérovingiens et des premiers royaumes francs. Les grands manuels d'histoire du XIX ${ }^{\mathrm{e}}$ siècle expédient en quelques lignes évasives ou lapidaires ce chapitre pourtant capital pour la destinée de la Gaule du sud et plus généralement de l'Empire romain d'Occident. Les Goths et leur entité politique, le regnum Tolosanum, n'ont pas, ou très peu, été intégrés aux grandes entreprises de construction d'une histoire nationale des deux derniers siècles. Les progrès de l'archéologie à la fin du XIX ${ }^{\mathrm{e}}$ siècle n'ont, hélas, pas bénéficié à cette problématique car le mobilier goth n'a pu être précisément caractérisé que bien plus tard. Les résultats des travaux pourtant amples et documentés de Casimir Barrière-Flavy (1892) sont à cet égard éloquents : une carte de géographie politique traitée comme une carte militaire [Fig.2] et des planches d'objets trouvés pour la plupart en contexte funéraire à l'intérieur de ce territoire [Fig.3], dont une faible minorité seulement s'avère appartenir effectivement à la culture matérielle des Goths. Depuis la seconde moitié $\mathrm{du} \mathrm{xx}^{\mathrm{e}}$ siècle cependant, plusieurs grandes études historiques (Loyen, 1934; Rouche, 1978 ; Treillet, 1984; Wolfram, 1990 ; Mathisen, Sivan, 1999, par exemple) ou archéologiques (Kazanski, 1991, 1995 ; Périn, Kazanski, 2011) ont beaucoup apporté à la connaissance de cette entité politique brutalement disparue.

Pour autant, le regard porté par les historiens sur les Goths de Gaule et leur «royaume de Toulouse» varie : les faits établis sont peu nombreux, et comme souvent l'interprétation des sources particulièrement délicate. Faut-il parler même parler de «royaume» (Delaplace, 2011) ? Sans doute, si les mots des Anciens avaient un sens.

Mais plus encore, l'appréciation par les historiens, depuis le XVIII ${ }^{\mathrm{e}}$ siècle, du rôle joué par les Goths de Gaule et d'Hispanie dans la désagrégation de l'Empire, est très variable ; elle le demeure jusqu'à aujourd'hui où le débat est parfois vif sur le thème : les Goths furent-il les derniers soutiens ou les fossoyeurs de l'autorité romaine? Trois réponses s'opposent : continuité (Goffart, 1980 ; Delaplace, 2010) ; rupture (Ward-Perkins, 2005 ; Barbero, 2009) ; abstention (Mathisen, Sivan, 1999).

La question était déjà posée par les historiens contemporains de l'installation des Goths, dont l'Espagnol Paul Orose, placé aux premières loges en 417. Plutôt favorable aux Goths et sévère quant aux actions du pouvoir romain, l'auteur déclare rapporter les propos d'un romain de Narbonnaise qui avait connu le premier roi Goth des Gaules, Athaulf ; en substance, ce dernier se serait pensé d'abord en fossoyeur, avant de se métamorphoser en soutien de Rome :

[Athaulfus,]...se inprimis ardenter inhiasse, ut, oblitterato Romano nomine, Romanum omne solum Gothorum imperium et faceret et uocaret, essetque, ut uulgariter loquar, Gothia quod Romania fuisset : fieret nunc A thaulfus quod quondam Caesar Augustus; at ubi multa experientia probauisset neque neque Gothos ullo modo parere legibus posse propter effrenatam barbariem neque reipublicae interdici leges oportere, sine quibus respublica non est respublica, elegisse saltim ut gloriam sibi de restituendo in integrum augendoque Romano nomine Gothorum uiribus quaereret habereturque apud posteros Romanae restitutionis auctor, postquam esse non potuerat immutator. "[Athaulf] avait avant tout ardemment aspiré, une fois effacé le nom romain, à faire de tout le territoire romain un empire des Gots et à l'appeler ainsi, et à ce que la Gotie, pour parler communément, fût ce que la Romanie avait été : à ce que lui, Athaulf, devînt alors ce que jadis 


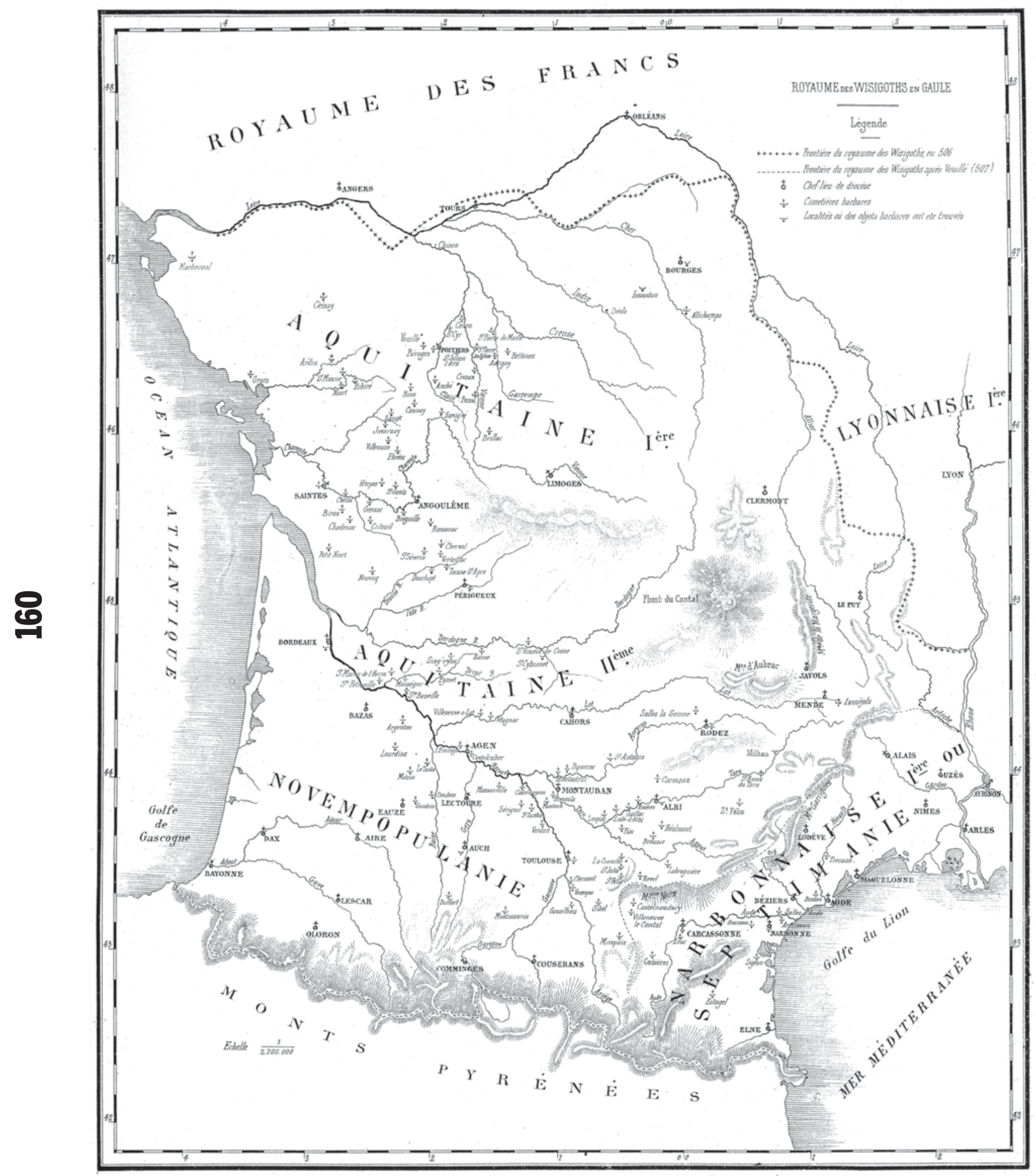

[Fig.2] Barrière-Flavy 1892. Carte du « royaume des Wisigoths", qui situe avec précision les « cimetières

barbares » et les « localités où des

objets barbares ont été trouvés ».

L'hydrographie, le relief ainsi que les

frontières administratives sont

figurés avec netteté, à l'instar de la

cartographie militaire de l'époque. 
avait été César Auguste ; mais quand une abondante expérience lui eut démontré que les Gots ne pouvaient en aucune manière obéir à des lois à cause de leur barbarie effrénée, et qu'il ne convenait pas à un état de supprimer les lois, lois sans lesquelles un état n'est pas un état, il avait choisi, à défaut, de rechercher pour lui la gloire de restaurer dans son intégralité et d'accroître le nom romain avec les forces des Gots, et d'être tenu dans la postérité pour l'auteur de la restauration de Rome, du moment qu'il n'avait pu en accomplir la mutation ${ }^{\mathbf{1}} »$.

La même année (417) pourtant, un haut fonctionnaire romain d'origine gauloise, Rutilius Namatianus (un Toulousain ?) écrit un poème sur son retour en Gaule, alors qu'il remonte les côtes de l'Italie ravagée par la guerre avec les Goths. Parlant de Rome, il déclame : Ergo age, sacrilegae tandem cadat hostia gentis /summittant trepidi perfida colla Getae/Ditia pacatae dent uectigalia terrae/impleat augustos barbara praeda sinus. «Courage donc! Qu'une nation sacrilège tombe enfin en victime! Que les Goths tremblants courbent devant toi leur cou perfide! Que leurs terres pacifiées te paient de riches tributs! Que le butin pris aux barbares emplisse ton sein auguste ${ }^{2}$ !»

Nous ne trancherons bien évidemment pas ici la question, nous bornant à présenter, dans l'ordre chronologique, des faits de nature historique ou archéologique qui nous paraissent signifiants pour notre approche : symptômes de processus de «déromanisation».

413-Aquitania Gothis tradita ${ }^{3}$ : «L'Aquitaine est abandonnée aux Goths ». Installation des Goths en Aquitaine manu militari, premier partage des terres, début de la prise de contrôle de l'administration impériale. En 412, deux ans après le sac de Rome et le pillage d'une grande partie de l'Italie, les Goths d'Althauf étaient intervenus en Gaule avec l'aval des autorités impériales et de certains milieux Gaulois ; il s'agit officiellement de débarrasser le pays des «tyrans » Jovin et Sebastien. C'est rapidement chose faite. Néanmoins, les nouveaux venus, hors de tout contrôle, ne tardent pas à s'emparer dès l'année suivante (413) de villes importantes entre les abords de la Méditerranée et la façade atlantique (Narbonne, Toulouse, Bordeaux...). Ils opèrent dans un pays désorganisé, que ce soit sur le plan social, économique ou militaire, par des années de

1 Orose, Histoires

(contre les païens), VII, 43, 5-6. Traduction

Marie-Pierre Arnaud-

Lindet, CUF, Les Belles

Lettres, Paris, 2003.

2 Rutilius Namatianus,

Sur son retour, I, 141-144.

Traduction Etienne

Wolff, CUF, Les Belles

Lettres, Paris, 2007.

3 L'Aquitaine est

abandonnée aux Goths.

Chronica Gallica a.

CCCCLII, année 413

4 Éd. Gundbach, M.G.H.

5 Hydace, Chronique,

année 418. Selon Prosber

Tiro, un Aquitain, en 419,

«Constantius patricius data ei ad inhabitandum

quibusdam civitatibus

confinium provinciarum»:

le patrice Constance ayant

ermi la paix avec Wallia

habiter, la seconde

Aquitaine et quelques

cités des provinces voisines.

Certains manuscrits

omettent «Secunda »,

alors que d'autres écrivent

«Aquitania ». pacem firmat cum Wallia, troubles : raids barbares, usurpations, guerres civiles. Le dispositif militaire romain en Gaule du Sud, auparavant peu développé si l'on prend en compte les données de la compilation de listes connue sous l'appellation de «Notitia Dignitatum», a disparu dans le chaos des années 407-412. De fait, l'armée disparue, aux termes de la loi romaine, les Goths sont seuls à porter les armes, ce qui n'a pu manquer de créer immédiatement un rapport de force aigu avec les populations présentes. Malheureusement, les sources littéraires sont elliptiques ou lapidaires sur la situation de la Gaule méridionale de 413 à 418 ; c'est pourtant durant cette période que durent se mettre en place les premiers processus de «déromanisation». Au premier chef, une appropriation quasi-immédiate par les Goths de terres et de biens selon des règles inusuelles en droit romain, fait indubitable au vu des sources juridiques gothes postérieures (Sivan, 1988 : Mathisen, Sivan, 1999). On ne sait pas exactement quelle était la zone contrôlée initialement par les Goths, au-delà de la vallée de la Garonne dont la possession est documentée par les sources contemporaines (Rouche, 1979); mais la liberté de mouvement des Goths a pu autoriser des gains territoriaux significatifs et rapides. Il est également probable que la haute administration civile romaine (ou ce qu'il en restait) ait été placée rapidement sous contrôle des autorités gothes : l'édit d'Honorius émis le 17 avril 418, qui rétablit à Arles le Conseil des Sept Provinces, stipule curieusement que la Novempopulanie et l'Aquitaine II sont des provinces «éloignées » et pourront envoyer des représentants subalternes si les magistrats sont «retenus ${ }^{\mathbf{4}}$ : ita ut de Novempopulana et secunda Aquitanica, quae provinciae longius constitutae sunt, si eorum iudices occupatio certa retenuerit, sciant, legatus iuxta consuitudinem esse mittendos. À notre connaissance, à compter de cette date, plus aucun haut fonctionnaire représentant le pouvoir civil impérial ne sera signalé dans les territoires tenus par les Goths.

418-419-Gothi (...) sedes in Aquitanica a Tolosa usque ad Oceanum acceperunt ${ }^{5}$ : «Les Goths (...) reçurent leur siège en Aquitaine, depuis Toulouse jusqu'à l'Océan ». Reconnaissance du pouvoir goth, délimitation de son assise territoriale par l'Empire et mise en place rapide d'une administration « royale». Après le succès des opérations militaires des Goths de Gaule en Espagne, officiellement pour le compte de l'Empire auxquels ils sont «alliés» depuis un traité passé en 382 , le patrice Constance, au nom de l'Empereur, leur concède en 418-419 un territoire dont ne connait pas la superficie (les termes du traité ne nous sont pas parvenus), mais qui pouvaient comporter une partie importante de l'ancienne «Aquitanique», notamment en direction de la Gaule centrale et de la Loire. Ceci dut aller rapidement de pair avec la mise en place d'une administration «royale» calquée sur celle de l'Empire. Ici intervient une découverte étonnante, faite à Bourges au XIX ${ }^{\mathrm{e}}$ siècle (Buhot de Kersers, 1892) : la tombe d'un personnage important,

(1)



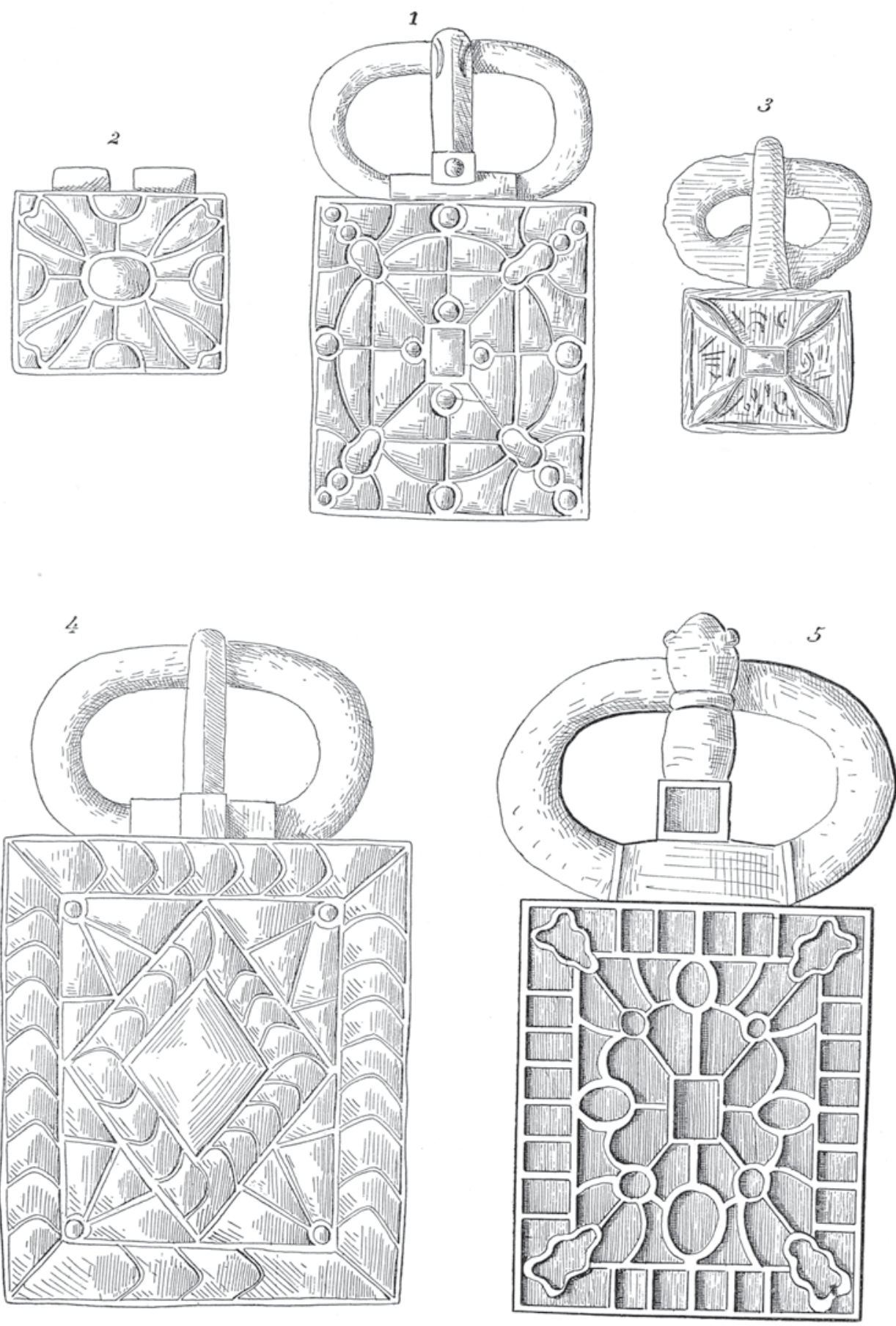

Prit, $S_{C}$.

C.B..F. del.

[Fig.3] Barrière-Flavy, 1892. " Agrafes de ceinturon ». Le travail du savant, basé sur un déterminisme géographique (c'est à dire les cimetières « barbares " situés à l'intérieur des limites du royaume wisigoth illustrent la présence de ce peuple), a été par la suite très décrié et son contenu négligé (à l'exception des superbes planches de mobilier, dont celle-ci qui figure d'authentiques plaquesboucles wisigothiques d'Aquitaine et de Narbonnaise, datant de la fin du $v^{\mathrm{e}}$ ou du début du vi ${ }^{\mathrm{e}}$ siècle). 
en habit et équipement militaire, dont une pointe de lance de parade portant l'inscription REGIUS PATRICIUS Z $(\text { ESES })^{\mathbf{6}}$ : «longue vie au Patrice du Roi !» (Bailly, 1984). Or parmi le mobilier d'accompagnement on note la présence d'une fiole de verre dont la datation n'est pas postérieure au début du $\mathrm{v}^{\mathrm{e}}$ siècle ; l'ensemble du mobilier, comme le type de tombe, incite à dater cette inhumation plusieurs décennies avant les années 469-507, comme cela est habituellement avancé. Si cette hypothèse se confirmait, cela impliquerait une présence gothe très précoce dans ces zones septentrionales. Les découvertes faites à Angers de tombes recelant des fibules wisigothiques anciennes pourraient aller dans le même sens (Brodeur et al., 2001). Les Goths étaient-ils déjà sur la Loire?

439-Inter Romanos et Gothos pax efficitur ${ }^{7}$ : "La paix est faite entre Goths et Romains". Foedereque firmato, ab alterutro pace peracta, recessit uterque : «On renouvela le traité, on se promit mutuellement de garder avec fidélité la paix, et l'on se retira de part et d'autre $»^{8}$. Nouveau traité sanctionnant l'échec de l'armée romaine. Contrôle par le roi goth du clergé catholique et des populations romaines.

Ces deux phrases laconiques concluent un épisode majeur qui lève le doute sur le degré de souveraineté déjà atteint par l'autorité gothe. Hydace parle de deux entités distinctes, les « Romains » (c'est à dire les représentants de l'autorité impériale en Gaule) et les «Goths», terme qui, dans le contexte, inclut les Romains du royaume goth. Une armée romaine vient d'être détruite au pied des murailles de Toulouse et son chef, Litorius, a été fait prisonnier et mourra en captivité. Une tentative de médiation avait été menée auparavant, à l'instigation du roi goth, qui a missionné Orens, évêque d'Auch en Novempopulanie. Le récit conservé dans sa Vita implique que l'évêque catholique ait été soumis au roi Goth ; c'est donc en tant que l'un de ses sujets qu'il tente une négociation infructueuse, face à l'autorité romaine (Bourdatchouk, Arramond, Grimbert, 2006 ; Bourdatchouk, 2009). La mainmise du roi gothsur le clergé catholique afférant au territoire qu'il contrôle (à l'image des pratiques de l'Empereur) est confirmée par Sidoine Apollinaire en 475 à la veille de la cession de l'Auvergne aux Goths (Epist. VII, 6) : l'évêque de Clermont constate avec amertume cette pratique ancienne et systématique à l'intérieur du territoire goth, qualifié (comme l'Empire !) de regnum, qu'il faut rendre par «puissance»; pour qualifier ce regnum, l'évêque emploie l'expression «limes Gothicae sortis » : les frontières de la zone gothe, et rappelle que les populations incluses dans cette zone échappent à l'autorité romaine.

451-453- «Dominus noster ... ». On note l'apparition sur certaines inscriptions romaines, notamment funéraires, du qualificatif «dominus noster» (notre maître), associé au nom du roi goth, la date étant établie au regard du nombre d'année de son règne, en lieu et place du nom de l'empereur

ou de la date consulaire? ${ }^{9}$. Les exemples sont nombreux en Aquitaine première, même en Auvergne (Prévôt 1997).

455- Le palatium des Goths. Une longue lettre de Sidoine Apollinaire, (Epist. I, 2) ${ }^{\mathbf{1 0}}$, écrite vers 455, nous renseigne sur l'édifice majeur de Toulouse gothe : le palais royal, alors occupé par Théodoric II. L'édifice est en relation avec un lieu de culte arien, sans doute réservé à la famille royale. Il est doté d'une salle du trône avec ses gardes exotiques et son décor aulique où le roi reçoit les gentium legationibus (légations des nations). On y trouve aussi une salle de banquet, des écuries, des appartements royaux et une salle du trésor qui font l'objet de mesures de sécurité particulières. Bref, le palais des rois goths rassemble l'essentiel des fonctions dévolues aux palais impériaux contemporains ; c'est l'expression d'un pouvoir souverain, perçu comme tel par Sidoine, alors présent en tant que « ressortissant étranger». Or l'archéologie préventive a permis, en 1990, de mettre au jour le long de la Garonne une partie d'un imposant complexe dont l'emprise minimale est de 100 sur 140 m (de Filippo, 1996). La chronologie de ce bâtiment est comprise dans le $\mathrm{v}^{\mathrm{e}}$ siècle. Les archéologues s'accordent aujourd'hui pour y reconnaître une partie du palais royal de Toulouse.

470-507- Des Romains dans l'armée gothe. Maximus ibi tunc Arvernorum populus, qui cum Apollinare venerat, et primi qui erant ex senatoribus, corruerunt: « un très grand nombre d'Arvernes qui étaient venus avec Apollinaire et qui étaient les premiers des sénateurs tombèrent alors » ${ }^{\mathbf{1 1}}$. C'est ainsi que Grégoire de Tours achève le récit de la bataille de Vouillé, qui voit la mort du roi Alaric II. Il n'y a pas lieu de douter de la véracité du fait : des Romains sont attestés dans l'armée du roi goth dès 474, au moment des troubles en Auvergne ${ }^{\mathbf{1 2}}$; c'est d'ailleurs un $d u x$ romain, Victorius, qui fut chargé des affaires militaires à l'issue de ce conflit.

475-506- Des lois pour les sujets du roi. Les deux recueils issus du royaume goth qui nous sont parvenus, le Code d'Euric et le Bréviaire d'Alaric, paraissent contrastés : le premier (très lacunaire) oppose les Romani et les Gothi et traite de procédures conflictuelles entre les deux communautés, dont le partage des terres ; le second (Rouche et Dumézil, 2008) est une adaptation du Code Théodosien, dont rien ne dit que l'usage était réservé aux Romains. Sans doute le roi avait-il voulu doter son royaume d'une législation propre, bien que proche des lois romaines en vigueur, à destination de tout son peuple.

Si l'on tente un bilan à partir de ces quelques faits, il parait évident qu'à la fin du processus, la «déromanisation» politique du royaume est accomplie et irréversible : les différents canaux par lesquels l'Empire administrait le territoire et les populations sont détruits, captés ou sous contrôle. La référence impériale a disparu. Le royaume goth, centré sur son roi, doté d'administrations à sa mesure inspirées du modèle romain, paraissait près de recréer,
6 Z Z ESES : translittération

7 Hydace, $C h t$

8 Jordan

sous Thorismond

(Wolfram 1990, p. 219).

Loyen, Lettres, II, (Livre

I à V), Paris, Les Belle

11 Grégoire de Tours,

Hist. Franc. II, 37

12 Sidoine Apollinaire

Epist. V, 12 
notamment au travers du droit et des institutions militaires, une sorte de « citoyenneté » au-delà des deux peuples en voie de rapprochement. Des processus que l'on avait vu à l'œuvre, initiés par Rome, quelques siècles auparavant....

BARRIÈRE-FLAVY C., 1892 : Étude sur les sépultures barbares du Midi et de l'Ouest de la France. Industrie wisigothique, Toulouse-Paris. Drioux ET Leroy, 1875 : Atlas universel et classique de géographie ancienne, romaine, du Moyen Age, moderne et contemporaine, Paris.

BARBERO A., 2009 : Barbares. Immigrés, réfugiés et déportés dans l'Empire romain, Tallandier, coll. Antiquité, Paris, $351 \mathrm{p}$.

BAILly P., 1984: «Une mention de « Patrice » dans une sépulture du $\mathrm{V}^{\mathrm{e}}$ siècle à Bourges ", in Actes du XLIII Congrès de la Fédération des sociétés savantes du Centre-Etudes creusoises V, Guéret, p. 39-43.

BOUDARTCHOUK J.-L., 2009: "Toulouse, de la ville wisigothique à la ville franque ( $\mathrm{v}^{\mathrm{e}}$-viII ${ }^{\mathrm{e}}$ siècle). Histoire et archéologie», in Toulouse, une métropole méridionale : vingt siècles de vie urbaine, Toulouse, éd. Méridiennes/Fédération historique de Midi-Pyrénées, vol. 1, p. 31-47.

Boudartchouk J.-L., Arramond J.-C., Grimbert L., 2006 : «Une sépulture de relégation découverte dans un fossé antique sur le site de l'Ilôt Castelbou (Toulouse, 1991) : un guerrier Vandale inhumé à la hâte? ", De l'âge du Fer au haut Moyen Age. Archéologie funéraire, princes et élites guerrières, t. XV des Mémoires de l'A.F.A.M., Condésur-Noireau, p. 122-133.

Brodeur J., Mortreau M., YvineC J.-H., 2001 : «Présence d'auxiliaires sur le site d'Angers. Mise en persbective au travers des fouilles d'Angers», in Militaires «barbares» dans l'armée romaine en Occident. Actualités archéologiques du haut Moyen Âge dans l'Ouest de la France, Pré-actes des XXII journées internationales d'archéologie mérovingienne, Angers, p. 9-12.

BUHOT DE KERSERS, 1892 : «Sépulture mérovingienne du cimetière des Capucins », in Mém. Soc. Ant. Centre, t. XVIII, 1891, Bourges, p. 51-63.

Demougeot E., 1979, La Formation de l'Europe et les invasions barbares, vol 2 : De l'avènement de Dioclétien au début du vi siècle, Aubier, Paris, $520 \mathrm{p}$.

De FILIPPO R., 1996 : « Le grand bâtiment du site de Larrey : la question palatiale», in Aquitania, XIV, p. 23-29.

Delaplace Ch., 2010 : «Le royaume wisigothique de Toulouse », in Wisigoths et Francs autour de la bataille de Vouillé (507). Recherches sur le haut Moyen Age dans le Centre-Ouest de la France, Actes des XXII Journées internationales d'archéologie mérovingienne (Vouillé et Poitiers, 28-30 septembre 2007), éd. AFAM, SaintGermain-en Laye, p. 15-18.

GofFART W., 1980 : Barbarians and Romans A. D. 418-584: the Techniques of Accommodation, Princeton University Press, Princeton, $279 \mathrm{p}$

KAZANSKI M., 1991 : Les Goths ( $I^{e r}-V I I^{e}$ après J.-C.), Paris, éd. Erance.

KAZANSKI M., LAPART J., 1995 : «Quelques documents du v ${ }^{\mathrm{e}}$ siècle ap. J.-C. attribuables aux Wisigoths découverts en Aquitaine», in Aquitania, XIII, p. 193-202.

LABrousse M., 1968 : Toulouse antique des origines à l'établissement des Wisigoths, Paris, École Française de Rome, 212.

LOYEN A., 1934: «Les Débuts du royaume wisigoth de Toulous », Revue des études latines, p. 406-415.

Mathisen R. W., 1999: Sivan H.S., «Forging a New Identity : the Kingdom of Toulouse and the Frontiers of Visigothic Aquitania (418-507)", in The Visigoths. Studies in Culture and Society, FERrEIro A. (ÉD.), Leiden-Boston-Köln, p. 2-62.

PÉRIN P., KAZANSKI M., 2011 : «Identity and Ethnicity during the Era of Migrations and Barbarian Kingdoms in the Light of Archaeology in Gaul», in Romans, Barbarians and the Transformation of the Roman World, Mathisen R.W. et Shanzer D. (éd.), Ashgate, p. 299-329.

PRÉVÔT F., 1997 : Recueil des inscriptions chrétiennes de la Gaule antérieures à la Renaissance carolingienne, t. 8 (Première Aquitaine), Paris, $168 \mathrm{p}$

Rouche M., 1979, L'Aquitaine des Wisigoths aux Arabes 418-781: naissance d'une nation, École des Hautes Études en Sciences Sociales, J. Touzot (éd.), Paris, 778 p.

Sivan H., 1984 : On Foederati, Hospitalitas and the settlement of the Goths in A.D. 418, in American Journal of Philology, vol. 108/4, p. 759-772.

Teillet S., 1984 : Des Goths à la nation gothique. Les origines de l'idée de nation en Occident du $V^{e}$ au VII siècle, coll. Études anciennes, Les Belles Lettres, Paris, 687 p.

WARD-PERKINS BR., The fall of Rome and the end of civilization, Oxford, 2005

Wolfram H., 1991, Histoire des Goths, coll. L'évolution de l'humanité, Albin Michel, Paris, 574 p.
L'archéologie préventive à la (re)découverte du peuple goth en Gaule du sud

\author{
Jean-Luc Boudartchouk \\ Jérôme Hernandez \\ Didier Paya \\ Christian Scuiller \\ Inrap
}

L es textes contemporains, si les mots latins qui nous sont parvenus ont un sens, sont sans ambiguïté sur un point : le peuple goth, qui prend possession de la Gaule du sud à partir de 413 et jusqu'en 508, constitue bien une entité sociologique et culturelle distincte au sein de l'ensemble de la population et son poids démographique, s'il est difficile à évaluer précisément, n'est pas négligeable. Or pendant longtemps les chercheurs ont eu du mal à caractériser de manière catégorique les vestiges archéologiques qui pouvaient leur être attribués, ce qui a conduit Edward James à conclure, en 1977, que sans les sources historiques la présence wisigothique en Gaule serait passée totalement inaperçue (James 1977). Jusqu'à une date récente, la majorité des chercheurs est restée fidèle à ce postulat, invoquant diverses raisons pour expliciter ce qui constitue tout de même un extraordinaire paradoxe : plus de cent mille, voire plusieurs centaines de milliers d'individus non romains, présents durant quatre générations en Gaule méridionale et centrale, n'auraient laissé aucune trace. On a évoqué (en minorant très fortement les chiffres donnés par les sources antiques) leur faible nombre au regard de la population romaine des provinces d'Aquitaine et de Narbonnaise. On a mis en avant l'état d'acculturation avancé des Goths à leur arrivée en Gaule : leur parcours chaotique dans le barbaricum puis à travers l'Empire romain d'est en ouest, les aurait peu à peu transformé en une armée errante (Wolfram, 1990). Cette mutation se serait accomplie en une seule génération, et serait conjointe à une transformation radicale de la structure même de la population (Kazanski 2010), tant au niveau de sa composition biologique que de sa stratification sociale (abandon d'éléments caractéristiques des sociétés germaniques non romanisées). Cette acculturation se serait accentuée pendant leur installation en Gaule : leur statut de militaires au service de l'Empire les aurait exclus des activités économiques et les aurait «obligés » à user d'objets de la vie quotidienne produits par les artisans locaux et à habiter dans les bâtiments construits selon des savoir-faire locaux. De manière générale, la plupart des historiens, hormis quelques-uns (Rouche, 1979), ont prêté aux Goths la volonté (au moins apparente) de se fondre dans la romanité.

Les archéologues ont eu tendance à partir de ces postulats pour interpréter les rares vestiges attribuables aux Goths. Cette présumée perméabilité aux influences romaines transparaitrait tout particulièrement au travers des pratiques funéraires, quasi absentes des textes mais révélées de manière spectaculaire par l'archéologie. Les Goths semblent 